\title{
Euler-Lagrange optimization of electric drives with DTM method
}

\author{
Pavol Oršanský ${ }^{1, *}$, Branislav Ftorek $^{1}$, Helena Šamajová ${ }^{1}$, Ján Vittek $^{2}$ \\ ${ }^{1}$ Department of Applied Mathematics, Faculty of Mechanical Engineering, University of Žilina, Univerzitná 1, \\ 01026 Žilina, Slovakia \\ ${ }^{2}$ Department of Electrical Engineering, Faculty of Electrical Engineering, University of Žilina, Univerzitná 1, \\ 01026 Žilina, Slovakia
}

\begin{abstract}
Euler-Lagrange optimization method is exploited here to develop energy saving position control strategy valid for a.c. drives. The optimization principle respects copper losses minimization only. Boundary value problem for EulerLagrange equations is solved by Differential transformation method (DTM).
\end{abstract}

Keywords: DTM-method, Euler-Lagrange equations, boundary value problem

\section{Introduction}

The consumption of electric motors represents nearly one half (46\%) of global electricity consumption. Therefore, even small decrease of energy demands of electric drives, can result in significant energy savings with subsequent contribution to the protection of the environment. The main contribution of this paper is a mathematical analysis of the energy optimal speed and position control of the drives with a permanent magnet synchronous motor (PMSM), taking into account stator copper losses. In $[1,2]$ the optimal mathematical trajectory is modified to correspond to the triangular and trapezoidal profiles. This procedure is necessary for the technical realization of the near-energy optimal control to any controlled drive with PMSM. The mathematical theory of optimization methods may be found in [3].

If we consider some functional and the fundamental problem: To determine the control function $\boldsymbol{u}(t)$ for which

$$
I_{0}=\int_{t_{A}}^{t_{B}} L_{0}(\mathbf{x}, \mathbf{u}, t) d t \rightarrow \min .
$$
[4].

The problem of plastic deformation considered due the minimization of total energy in

\footnotetext{
*Corresponding author: pavol.orsansky@fstroj.uniza.sk

Reviewers: Tomáš Lack, Jerzy Winczek
} 
The function $L_{0}$ describes a minimization problem with the constraints (equations of motion for some physical problem, e.g. Newton's eq. of motion $F=m \mathbf{a}$ )

$$
\frac{d \mathbf{x}}{d t}=f(\mathbf{x}, \mathbf{u}, t)
$$

and the complete cost functional is defined by the relation

$$
\mathfrak{I}=L_{0}(\mathbf{x}, \mathbf{u}, t)+\lambda^{T}\left[\frac{d \mathbf{x}}{d t}-f(\mathbf{x}, \mathbf{u}, t)\right],
$$

where $\lambda^{T}$ is a vector of Lagrange multipliers. The solution of the minimizing problem requires to find a solution of Euler-Lagrange equations with some boundary conditions

$$
\frac{d \mathfrak{I}}{d \mathbf{x}}=\frac{d}{d t}\left(\frac{\partial \mathfrak{I}}{\partial \dot{\mathbf{x}}}\right), \quad \frac{d \mathfrak{I}}{d \mathbf{u}}=\frac{d}{d t}\left(\frac{\partial \mathfrak{I}}{\partial \dot{\mathbf{u}}}\right) .
$$

By means of Euler-Lagrange equations we can find the optimal control laws for electrical drive system.

Following differential equations describe dynamics of the rotor position $\phi$, speed $\omega$, electrical motor torque $\gamma_{e}$ and load torque $\gamma_{l}$

$$
\begin{gathered}
\frac{d \phi}{d t}=\omega, \\
J_{r} \frac{d \omega}{d t}=\gamma_{e}-\gamma_{l}, \\
\frac{d \gamma_{e}}{d t}=-\frac{1}{T_{S}} \gamma_{e}-\frac{k_{t}^{2}}{L_{S}} \omega+\frac{k_{t}}{L_{S}} u_{q},
\end{gathered}
$$

where $J_{r}$ is the reduced moment of an inertia to the shaft PMSM, $L_{S}$ and $R_{S}$ are stator phase inductance and resistance respectively, $T_{S}=\frac{R_{S}}{L_{S}}$ is a stator time constant. The electrical torque of the motor can be expressed as

$$
\gamma_{e}=k_{t} i_{q}
$$

where $k_{T}=c \psi_{P M}$ and $c=(3 p) / 2, p$ is a number of pol-pairs, $\psi_{P M}$ is a linkage flux of the permanent magnet and $i_{p}=p(t)$ is a torque component of the stator current.

An energy consumption of the control system depends on the minimization of copper losses and is given as (see [5])

$$
I_{0}=R_{S} \int_{t_{A}}^{t_{B}} i_{q}^{2} d t \rightarrow \min .
$$

With regard to (4), we have the relation

$$
I_{0}=\frac{R_{S}}{k_{t}^{2}} \int_{0}^{T_{m}} \gamma_{e}^{2} d t \rightarrow \min
$$

where $T_{m}$ is a manoeuvre time. If we assume that the load torque is a time dependent function $\gamma_{l}=f(t)$, then the complete cost functional (1) has a form (see also (3))

$$
\mathfrak{J}=\frac{R_{S}}{k_{t}^{2}} \gamma_{e}^{2}+\lambda_{1}\left(J_{r} \dot{\omega}-\gamma_{e}+\gamma_{l}\right)+\lambda_{2}(\dot{\phi}-\omega)+\lambda_{3}\left(\dot{\gamma}_{l}-\dot{f}(t)\right) \text {. }
$$


The corresponding Euler-Lagrange equations (2) for the position control are

$$
\begin{gathered}
2 \gamma_{e} \frac{R_{S}}{k_{t}^{2}}-\lambda_{1}=0, \\
-\lambda_{2}=J_{r} \dot{\lambda}_{1}, \\
0=\dot{\lambda}_{2}, \\
\lambda_{1}=\dot{\lambda}_{3} .
\end{gathered}
$$

According to (5), we have $\gamma_{e}=\frac{k_{t}^{2}}{2 R_{S}}$ and $\gamma_{l}=f(t)$ (the assumption). Finally, from Euler-Lagrange equations with constrains (3) follows a boundary value problem (BVP)

$$
\begin{gathered}
\dot{\lambda}_{1}=\frac{1}{J_{R}} \lambda_{2}, \\
\dot{\lambda}_{2}=0, \\
\dot{\omega}=\frac{1}{J_{R}}\left[\frac{k_{t}^{2}}{2 R_{S}} \lambda_{1}-f(t)\right], \\
\dot{\phi}=\omega .
\end{gathered}
$$

With conditions

$$
\begin{gathered}
\omega(0)=\omega\left(T_{M}\right)=0, \\
\phi(0)=0, \\
\phi\left(T_{M}\right)=\phi_{\text {dem }}
\end{gathered}
$$

where the manoeuvre time $T_{M}$ and the demanded position $\phi_{d e m}$ are given.

\section{DTM - Differential transformation method}

We give a brief overview of Differential transformation method that proposes a technique, which does not evaluate the derivatives symbolically, the DTM of the $n$-th derivative of a function $y(t)$ is defined by

$$
Y(n)=\frac{1}{n !}\left[\frac{d^{n} y(t)}{d t^{n}}\right]_{t=t_{0}}
$$

where $y(t)$ is an original and $Y(n)$ is a transformed function.

An inverse transformation of $Y(n)$ leads to

$$
y(t)=\sum_{n=0}^{\infty} Y(n)\left(t-t_{0}\right)^{n}
$$

and if we consider $t_{0}=0$ then the representation of the function reduces to

$$
y(t)=\sum_{n=0}^{\infty} Y(n) t^{n} .
$$

The properties of the DTM with their proofs are published in [6, 7], the used ones of them are introduced below.

Let functions $G, G_{i}$, for $i=1,2, \ldots, N$ be differential transformations of functions $g, g_{i}$, for $i=1,2, \ldots, N$, then the following correspondences hold

$$
g(t)=g_{1}(t) \pm g_{2}(t), \quad G(n)=G_{1}(n) \pm G_{2}(n),
$$




$$
\begin{array}{cc}
g(t)=\alpha g_{1}(t), & G(n)=\alpha G_{1}(n), \\
g(t)=g_{1}(t) \cdot g_{2}(t), & G(n)=\sum_{l=0}^{\infty} G_{1}(n) \cdot G_{2}(n-l), \\
g(t)=t^{k}, & G(n)=\delta_{n, k}, t_{0}=0, \\
g(t)=\frac{d^{k}}{d t^{k}} g_{1}(t) & G(n)=\frac{(n+k) !}{n !} G_{1}(n+k), \\
g(t)=\prod_{i=1}^{N} g_{i}(t), & \\
G(n)=\sum_{s_{1}=0}^{n} \sum_{s_{2}=0}^{n} \ldots \sum_{s_{N}=0}^{n-s_{1}-\ldots-s_{N-1}} G_{1}\left(s_{1}\right) \cdot \ldots \cdot G_{N}\left(n-s_{1}-\cdots-s_{N}\right),
\end{array}
$$

where $\delta_{n, k}$ is the Kronecker delta symbol, $n, l, k, N \in \mathbb{Z}_{0}^{+}$.

\section{The BVP solution using DTM method}

The standard using of the DTM transform is given for initial value problems and the solution has a form (8). In the next, we derive an approximate solution of the BVP problem (6), (7) by using some properties of DTM method. So, we have

$$
\begin{gathered}
\lambda_{1} \rightarrow U_{1}(n), \lambda_{2} \rightarrow U_{2}(n), \omega \rightarrow U_{3}(n), \phi \rightarrow U_{4}(n), f(t) \rightarrow F(n) \\
(n+1) U_{1}(n+1)=-\frac{1}{J_{r}} U_{2}(n), \\
(n+1) U_{2}(n+1)=0, \\
(n+1) U_{3}(n+1)=\frac{1}{J_{r}}\left[\frac{k_{t}^{2}}{2 R_{S}} U_{2}(n)-F(n)\right], \\
(n+1) U_{4}(n+1)=U_{3}(n) .
\end{gathered}
$$

In praxis, the manoeuvre time $T_{m} \leq 1 s$ and we consider the approximate solution in the form, i.e. the polynomials of the third degree

$$
\begin{aligned}
\lambda_{1} & =U_{1}(0)+U_{1}(1) t+U_{1}(2) t^{2}+U_{1}(3) t^{3}, \\
\lambda_{2} & =U_{2}(0)+U_{2}(1) t+U_{2}(2) t^{2}+U_{2}(3) t^{3}, \\
\omega & =U_{3}(0)+U_{3}(1) t+U_{3}(2) t^{2}+U_{3}(3) t^{3}, \\
\phi & =U_{4}(0)+U_{4}(1) t+U_{4}(2) t^{2}+U_{4}(3) t^{3} .
\end{aligned}
$$

Technically, from (9) we have 12 linear equations $(n=0,1,2)$ for 16 unknown coefficients $U_{i}(k), i=1,2,3,4$ and $i=0,1,2,3$. The other four linear equations we get from the boundary conditions (7), i.e.

$$
\begin{gathered}
U_{3}(0)=0 \\
U_{3}(1) T_{m}+U_{3}(2) T_{m}^{2}+U_{3}(3) T_{m}^{3}=0 \\
U_{4}(0)=0 \\
U_{4}(1) T_{m}+U_{4}(2) T_{m}^{2}+U_{4}(3) T_{m}^{3}=\phi_{d e m}
\end{gathered}
$$




\section{Numerical results}

The parameters of the numerical calculation are: $R_{S}=1.3 \Omega, k_{T}=0.975$, the moment of inertia $J_{r}=0.005 \mathrm{~kg} \cdot \mathrm{m}^{2}$, the prescribed manoeuvre time and the demanded position are $T_{m}=0.5 \mathrm{~s}, \phi_{\text {dem }}=2 \pi$. We assume two cases of the load torque $\gamma_{l}$

constant

$$
\gamma_{l}=2 \text {, }
$$

exponential

$$
\gamma_{l}=2+e^{2 t}
$$

The corresponding energy optimal solutions for these particular problems are for $\gamma_{l}=2$,

$$
\begin{gathered}
\omega(t)=150.7964 t-301.5929 t^{2} \\
\phi(t)=75.3982 t^{2}-100.531 t^{3} .
\end{gathered}
$$

and for $\gamma_{l}=2+e^{2 t}$

$$
\begin{gathered}
\omega(t)=84.1298-101.5929 t^{2}-133.3333 t^{3}, \\
\phi(t)=42.0649 t^{2}-33.8643 t^{3} .
\end{gathered}
$$

Results for energy optimal control of the drive speed and the drive position in the case of the constant load torque $\gamma_{l}=2$, are shown in Figure 1 (respectively for the exponential load torque $\gamma_{l}=2+e^{2 t}$ in Figure 2)

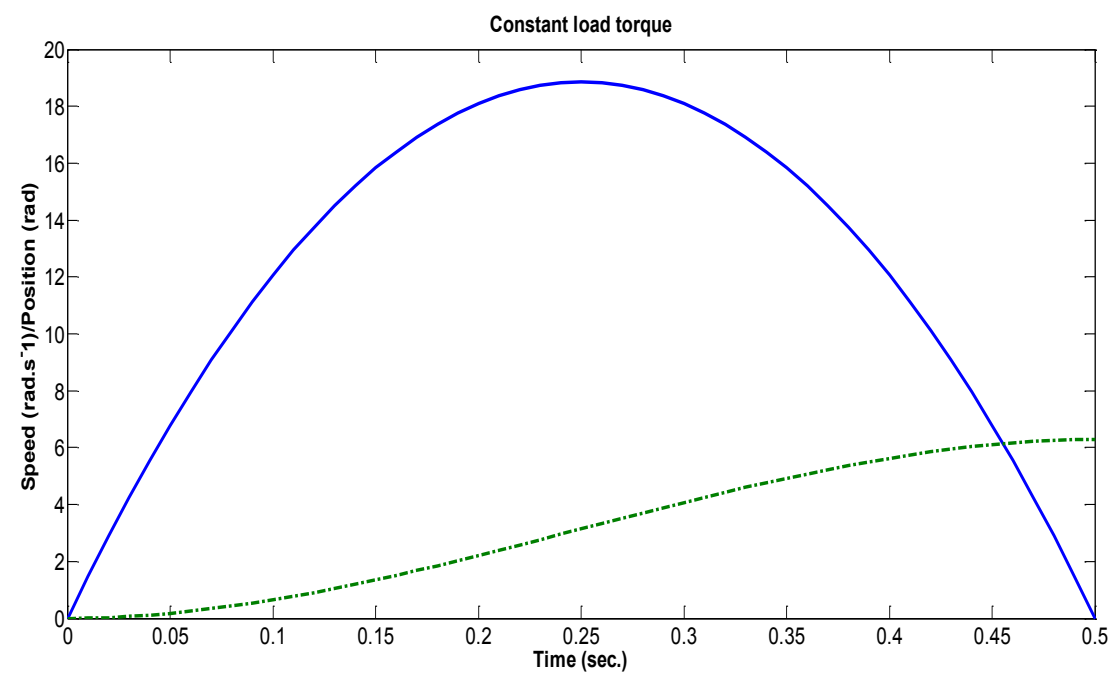

Fig. 1. Energy optimal control of the drive speed and the drive position in the case of the constant load torque $\gamma_{l}=2$ (speed $\omega(t)$ - blue line, position $\phi(t)$ dash-dot line). 


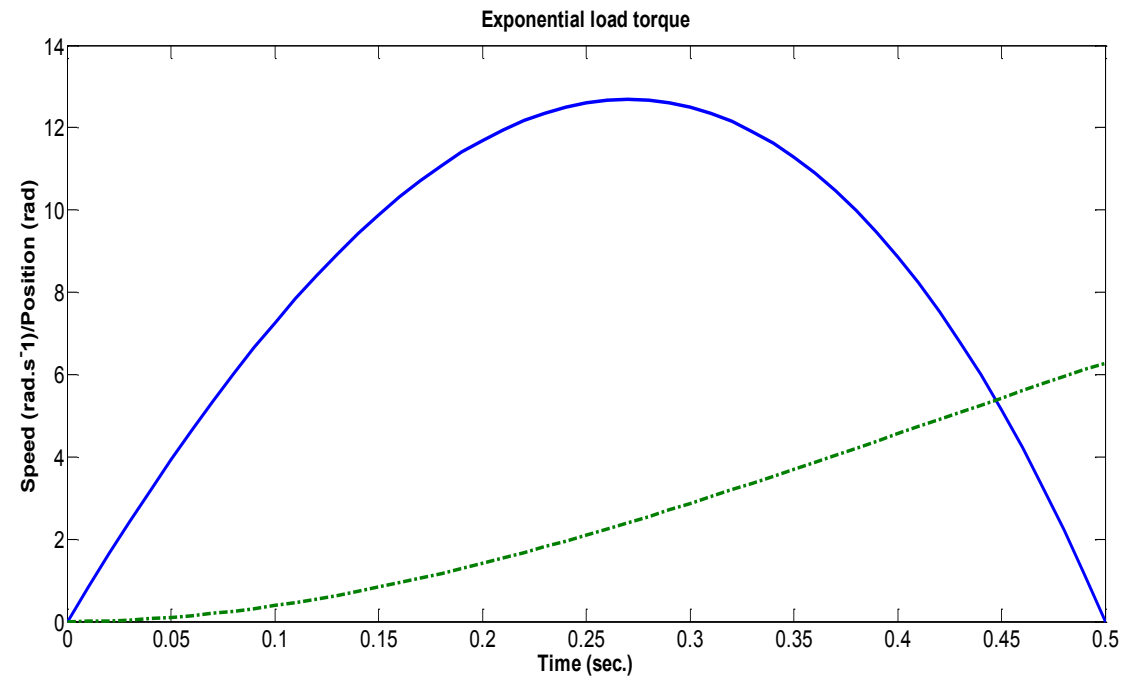

Fig. 2. Energy optimal control of the drive speed and the drive position in the case of the exponential load torque $\gamma_{l}=2+e^{2 t}$ (speed $\omega(t)$ - blue line, position $\phi(t)$ - green dash-dot line).

\section{Conclusion}

In this paper the Differential transformation method is used to solve linear system of differential equations derived for optimal speed and position control of the drives with a permanent magnet synchronous motor (PMSM), taking into account stator copper losses. This problem needs to solve Euler-Lagrange equations with some constraints and boundary value conditions. The different types of load torques are considered, namely the constant and exponential load torques. The approximate solutions of BVP are given in the form of series with required accuracy, some numerical examples are provided.

The authors gratefully acknowledge the Scientific Grant Agency (KEGA) of the Ministry of Education of Slovak Republic for supporting this work under the Grant No. 015ŽU-4/2017 Digital technologies for machine building study programs as a part of strategy "Internet of Things"

\section{References}

1. J. Vitek, B. Ftorek: Energy efficient speed and position control of electric drives with PMSM, Communications. Scientific letters of the University of Žilina 16, 64-71 (2014)

2. J. Vitek, B. Ftorek, P. Butko, T. Fedor: Energy optimal control of PMSM Drive for time-varying load torque, Applied mechanics and materials 710, 67-75 (2015)

3. H. Tolle: Optimization Methods, Springer-Verlag, New-York, (1975)

4. J. Zapoměl, V. Dekýš, P. Ferfecki, A. Sapietová, M. Sága, M. Žmindák: Identification of Material Damping of a Carbon Composite Bar and Study of Its Effect on Attenuation of Its Transient Lateral Vibrations. International Journal of Applied Mechanics 7, 1-18 (2015)

5. I. Bivol, C. Vasilache: The application of Euler-Lagrange method of optimization for electromechanical motion control. The Annals of Dunarea De Jos University of Galati III, 5-11 (2000) 
6. A. Arikoglu, I. Özkol: Solution of difference equations by using differential transform method. Appl. Math. Comput. 174, 1216-1228 (2006)

7. Y. Khan, Z. Svoboda, Z. Šmarda: Solving certain classes of Lane-n type equations using differential transformation method. Advances in difference equations 2012:174, 1-11 (2012) 